\title{
Lipid Mediator n-3 Docosapentaenoic Acid-Derived Protectin D1(PD1n-3DPA) Enhances Synaptic Inhibition of Hippocampal Principal Neurons by Interaction with a G-Protein Coupled Receptor
}

Apostolos Mikroulis ( $\square$ apostolos.mikroulis@med.lu.se)

Lunds Universitet https://orcid.org/0000-0002-3530-8895

Marco Ledri

Lunds Universitet

Gabriele Ruffolo

Sapienza University of Rome: Universita degli Studi di Roma La Sapienza

Eleonora Palma

Sapienza University of Rome: Universita degli Studi di Roma La Sapienza

Günther Sperk

Medizinische Universität Innsbruck: Medizinische Universitat Innsbruck

Jesmond Dalli

Queen Mary University of London

Annamaria Vezzani

Istituto di Ricerche Farmacologiche Mario Negri

Merab Kokaia

Lunds Universitet

\section{Research Article}

Keywords: PD1n-3DPA, GABAA receptors, perisomatic inhibition, mouse hippocampus, antiepileptic mechanism

Posted Date: September 16th, 2021

DOI: https://doi.org/10.21203/rs.3.rs-667784/v1

License: (c) (1) This work is licensed under a Creative Commons Attribution 4.0 International License. Read Full License 
Lipid mediator n-3 docosapentaenoic acid-derived protectin D1(PD1 $1_{n-3 D P A}$ ) enhances synaptic inhibition of hippocampal principal neurons by interaction with a G-protein coupled receptor

Apostolos Mikroulis ${ }^{1}$, Marco Ledri ${ }^{1}$, Gabriele Ruffolo ${ }^{2,3}$, Eleonora Palma ${ }^{2}$, Günther Sperk ${ }^{4}$, Jesmond Dalli $^{5}$, Annamaria Vezzani ${ }^{6}$, Merab Kokaia $^{1,7}$

1 - Epilepsy Center, Department of Clinical Sciences, Faculty of Medicine, Lund University, Sweden

2 - Department of Physiology and Pharmacology, Istituto Pasteur-Fondazione Cenci Bolognetti, University of Rome Sapienza, Rome, Italy

3 - IRCCS San Raffaele Pisana, Rome, Italy

4 - Department of Pharmacology, Medical University Innsbruck, Austria

5 - William Harvey Research Institute, Barts and The London School of Medicine and Dentistry, Queen Mary University of London, UK

-Centre for Inflammation and Therapeutic Innovation, Queen Mary University of London, UK

6 - IRCCS-Mario Negri Institute for Pharmacological Research, Milan, Italy

7 - Corresponding author: Merab.Kokaia@med.lu.se 


\section{Abstract}

Epilepsy is a severe neurological disease manifested by spontaneous recurrent seizures due to abnormal hyper-synchronisation of neuronal activity. Epilepsy affects about $1 \%$ of the population and up to $40 \%$ of patients experience seizures that are resistant to currently available drugs, thus highlighting an urgent need for novel treatments. In this regard, anti-inflammatory drugs emerged as potential therapeutic candidates. In particular, specific molecules apt to resolve the neuroinflammatory response occurring in acquired epilepsies have been proven to counteract seizures in experimental models, and in humans. One candidate investigational molecule has been recently identified as the lipid mediator $n-3$ docosapentaenoic acid-derived protectin D1(PD1 $n$-3DPA) which significantly reduced seizures, cell loss and cognitive deficit in a mouse model of acquired epilepsy. However, the mechanisms that mediate PD1 $1_{n-3 D P A}$ effect remain elusive. We here addressed whether PD1 $1_{n-3 D P A}$ has direct effects on neuronal activity independent on its anti-inflammatory action. We incubated therefore hippocampal slices with PD $1_{\text {n-3DPA }}$ and investigated its effect on excitatory and inhibitory synaptic inputs to the CA1 pyramidal neurons. We demonstrate that inhibitory drive onto the perisomatic region of the pyramidal neurons is increased by PD1 $1_{n-3 D P A}$, and this effect is mediated by pertussis toxin-sensitive G-protein coupled receptors. Our data indicate that PD $1_{n-3 D P A}$ acts directly on inhibitory transmission, most likely at presynaptic site of inhibitory synapses as also supported by oocyte and immunohistochemical experiments. Thus, in addition to its anti-inflammatory effects, PD1 $1_{\text {-3DPA }}$ anti-seizure and neuroprotective effects may be mediated by its direct action on neuronal excitability by modulating their synaptic inputs.

\section{Declarations}

\section{Funding}

AM, MK, were supported by European Union's Seventh Framework Programme (FP7/2007-2013) under Grant Agreement n602102, Swedish Research Council grant No. 2017-00921, and Olle Engkvists Foundation, Sweden.

GS Austrian Science Fund (Project P 19464), technical help by Anna Wieselthaler-Hoelzl GR was supported by BE-FOR-ERC program (Sapienza University) and Italian Ministry of Health "Ricerca corrente" 
JD is supported by a Sir Henry Dale Fellowship jointly funded by the Wellcome Trust and the Royal Society (grant\# 107613/Z/15/Z).

AV, was supported by European Union's Seventh Framework Programme (FP7/2007-2013) under Grant Agreement n602102.

\section{Conflicts of interest/Competing interests}

The authors have no relevant financial or non-financial interests to disclose.

\section{Availability of data and material}

The datasets generated during and/or analysed during the current study are available from the corresponding author on reasonable request.

\section{Code availability}

Code is available at GitHub and Zenodo with DOI: $10.5281 /$ zenodo.4431620

\section{Authors' contributions}

Conceptualization: MK, AV, JD; Methodology: MK, AV, ML, AM, GS, EP, ...; Formal analysis and investigation: $A M, M L, G R, E P, G S$; Writing - original draft preparation: AM; Writing - review and editing: AM, ML, GRR, EP, GS, JD, AV, MK; Resources: EP, GS, JD, MK; Supervision: MK

\section{Ethics approval}

All experiments were approved and conducted according to the Lund/Malmö experimental animal committee ethical permits 02998/2020 - M47-15.

\section{Keywords}

$\mathrm{PD}_{\mathrm{n}-3 \mathrm{BPA}}, \mathrm{GABA}_{\mathrm{A}}$ receptors, perisomatic inhibition, mouse hippocampus, antiepileptic mechanism

\section{Introduction}

Epilepsy is one of the most common and devastating neurological disorders affecting about $1 \%$ of the world population. Available antiseizure drugs (ASDs) are mainly providing symptomatic relief and fail to adequately control seizures in about $40 \%$ of patients. Alternative treatments include surgical removal of seizure focus, vagal nerve and deep brain stimulations, and dietary supplementation such as ketogenic or PUFAs-enriched diet [1]. However, these treatments offer variable efficacy outcomes and surgery can be proposed only to a restricted number of patients [2]. Therefore, there is a high 
demand to develop novel treatments for epilepsy based on a better knowledge of the pathogenic mechanisms underlying seizures.

Neuroinflammation has been described as a common pathogenic mechanism promoting seizures in animal models of acquired epilepsy, and in common forms of human drug-resistant epilepsy [3]. Pharmacological interventions with specific anti-inflammatory drugs provided evidence in support of a pivotal involvement of cytokines, chemokines, complement factors and prostaglandins, in seizure generation and recurrence [4]. Notably, inflammatory molecules contribute to increase neuronal network excitability by various mechanisms, which include modulation of synaptic transmission $[5,6]$, changes in blood brain barrier (BBB) permeability and consequent excitatory synaptogenesis [7], and alterations in glial cell function ([8]. Altogether, these changes lead to pathophysiological deregulation of neuronal networks promoting hyper-synchronisation and seizures $[3,5,9]$. It has been proposed that neuroinflammation in epilepsy persists due to inefficient engagement of resolving mechanisms, as supported by evidence that anti-inflammatory agents decrease seizures by reducing neuroinflammation [3].

Endogenous pro-resolving systems operate both in peripheral tissue and CNS and include various proteins [3] and key lipid mediators derived from n-3 PUFAs such as lipoxins, resolvins, protectins and maresins [10]. One such resolving molecule has been recently identified by some of us as $n-3$ docosapentaenoic acid-derived protectin D1 (PD1 $\left.1_{n-3 D P A}\right)$, which increases to insufficient extent in the hippocampus of mice developing epilepsy [11]. In fact, supplementation of PD1 $1_{n-3 D P A}$ during epilepsy development by its intracerebroventricular injection, reduced recurrent spontaneous seizures as well as mRNA levels of IL-1 $\beta$ and TNF ictogenic cytokines. Comorbidities, such as weight loss and impaired cognitive function were also ameliorated. Although resolution of neuroinflammation by PD $1_{n-3 D P A}$ is likely involved in its therapeutic effects, this lipid mediator might also affect network excitability and synaptic transmission by yet unknown direct mechanisms. In support, there is evidence that n-3 PUFAs (such as EPA, DHA) decrease evoked hippocampal neuronal excitability in brain slices [12, 13]. 
To address this hypothesis, we incubated naïve mouse hippocampal slices with $\mathrm{PD} 1_{\mathrm{n}-3 \mathrm{DPA}}$ and investigated its effect on excitatory and inhibitory synaptic drive onto the pyramidal neurons of CA1 region. We demonstrate that $\mathrm{PD} 1_{\mathrm{n}-3 \mathrm{DPA}}$ increases inhibitory synaptic drive onto the pyramidal neurons mediated by GPCRs, and thereby may counteract the increased excitability of hippocampal network resulting in seizure inhibition. 


\section{Methods}

Animals

Mice with C57/BL6 background were bred and housed (5 mice/cage) in the SPF facility at a constant room temperature $\left(23^{\circ} \mathrm{C}\right)$ and relative humidity $(60 \pm 5 \%)$ with free access to food and water, and with a fixed $12 \mathrm{~h}$ light/dark cycle. All experiments were approved and conducted according to the Lund/Malmö experimental animal committee ethical permits 02998/2020 - M47-15.

\section{PD1 $1_{n-3 D P A}$ solution preparation}

For electrophysiology in slices or oocytes, as well as for GABAR immunohistochemistry, the PD1 $1_{\text {n-3DPA }}$ (10 $\mathrm{mM}$ in ethanol) was kept at $-80^{\circ}$ until the experiment was started. Immediately before the incubation, the ethanol was evaporated with nitrogen gas and the compound was solubilized in 450 $\mu \mathrm{l}$ artificial cerebrospinal fluid (aCSF) for experiments in slices (118 mM NaCl, $2 \mathrm{mM} \mathrm{MgCl}, 2 \mathrm{mM}$ $\mathrm{CaCl}_{2}, 2.5 \mathrm{mM} \mathrm{KCl}, 26 \mathrm{mM} \mathrm{NaHCO}_{3}, 1.25 \mathrm{mM} \mathrm{NaH}_{2} \mathrm{PO}_{4}$, and $10 \mathrm{mM} \mathrm{D-glucose)}$ or in Barth's solution (88 mM NaCl, $1 \mathrm{mM} \mathrm{KCl}, 2.4 \mathrm{mM} \mathrm{NaHCO}{ }_{3}, 10 \mathrm{mM} \mathrm{HEPES}, 0.82 \mathrm{mM} \mathrm{MgSO}_{4}, 0.33 \mathrm{mM} \mathrm{Ca}\left(\mathrm{NO}_{3}\right)_{2}, 0.41$ $\mathrm{mM} \mathrm{CaCl}{ }_{2}$ ) for experiments in oocytes to yield a $10 \mathrm{nM}$ solution, which was instantly used.

\section{Mouse hippocampal slice electrophysiology}

After weaning, 3-10 weeks-old mice were anaesthetised with isoflurane, sacrificed, and the brains were quickly removed and subsequently processed in ice-cold aCSF. The cerebellum was removed, and $300 \mu \mathrm{m}$ horizontal slices were cut with a Leica $1200 \mathrm{~S}$ VT vibratome, typically between $900 \mu \mathrm{m}$ to $1800 \mu \mathrm{m}$ from the ventral part of the brain.

Two to 3 slices containing the ventral hippocampus were prepared for electrophysiology. The slices (containing the posterior part of both hemispheres) were further dissected into 2 smaller slices of the hippocampus and peri-hippocampal areas, with a sagittal-plane cut medially to the outer shell of the dentate gyrus (Figure 1). This was done to enable easier transport between chambers, reduce the glucose and oxygen demands, as well as metabolite accumulation during incubation in the limited volume chamber. 
From each animal, one or two slices (per condition) were incubated at room temperature $\left(20^{\circ} \mathrm{C}\right)$ for $1 \mathrm{~h}$ in a small volume interface-type chamber with $225 \mu \mathrm{L}$ of aCSF, either with $10 \mathrm{nM}$ PD1 $1_{\mathrm{n}-3 \mathrm{DPA}}$ or vehicle (aCSF). The $\mathrm{G}_{\mathrm{s}}$ protein antagonist pertussis toxin (PTX; Tocris), $1 \mu \mathrm{g} / \mathrm{mL}$ was added together with PD1 $n$-3DPA in a subset of slices.

\section{Hippocampal slice electrophysiology}

Glass pipettes were pulled from thick-wall Stoelting (ID/OD 0.75/1.50) or King Precision borosilicate glass (ID/OD 0.86/1.50) capillaries on a Sutter P-97 puller and filled with a pipette solution containing CsCl 135 mM, NaCl 8 mM, CsOH EGTA 0.2 mM, CsOH HEPES 10 mM, MgATP 2 mM, Na ${ }_{3}$ GTP 0.3 mM, QX-314 $5 \mathrm{mM}$ and $0.2 \%$ biocytin. Pipette resistance was within 2-5 MOhm.

Spontaneous and miniature post-synaptic currents (PSCs) were recorded at $10 \mathrm{kHz}$, after a $3 \mathrm{kHz}$ antialiasing Bessel filter, in gap-free mode, from mid-distal CA1 pyramidal neurons, voltage-clamped at $-70 \mathrm{mV}$, with either NBQX $5 \mu \mathrm{M} / \mathrm{AP} 550 \mu \mathrm{M}$ or PTX $100 \mu \mathrm{M}$ in the perfusion medium. A HEKA EPC9 and HEKA Patchmaster v13.52 for Apple macOs was used for acquisition.

Post-synaptic currents were detected with the correlation coefficient method [15] and analysed using Intel Python 3.6 under Windows 10 (code available at [16]. Events were selected if their correlation coefficient with a double exponential fit of averaged post-synaptic currents exceeded 0.6, with an amplitude greater than 3 pA (amplifier noise floor), 20-80\% rise time less than $5 \mathrm{~ms}$ and halfwidth greater than the rise time, to exclude potential artifacts.

After filtering, the following numbers of events were analysed. sIPSCs: $8 \mathrm{ctrl}$ and 9 PD1 $1_{n-3 D P A}$ incubated cells with 545 events each, mIPSCs: 5 ctrl and 8 PD $1_{n-3 D P A}$ incubated cells with 929 events each, sEPSCs: 9 ctrl and 9 PD1 $1_{\text {n-3DPA }}$ incubated cells with 646 events each, mEPSCs: 8 ctrl and 8 PD1n-3DPA incubated cells with 357 events each. For the rise time classification of fast and slow events, to ensure that we applied a threshold where ratio changes were approximately equal between groups and locally stable, thus not artificially inflating one of the categories, we applied the following steps: 
For all possible cut-off rise-time values, $\tau_{c}$ in $\delta \tau$ intervals, where $\delta$ denotes the minimum discrete difference,

$$
\tau_{c}=k \cdot \delta \tau, k \in \mathbb{N}, k>0, \delta \tau>0
$$

we calculate the ratio, $r_{s: f}$, of counts of observed rise-times slower-than to faster-than $\tau_{c}$,

$$
r_{s: f}=\frac{n_{\tau>\tau_{c}}+1}{n_{\tau \leq \tau_{c}}+1}
$$

and select the minimum $\tau_{c}$ where the rates of change of $r_{s: f}$ for the 2 groups are equal or approximately equal, after the $r_{s: f}$ spike at the beginning of the $\tau_{c}$ range:

$$
\widehat{\tau}_{c}=\min \left(\tau_{c}\right): r_{s: f} \ll \max \left(r_{s: f}\right), \frac{\delta r_{s: f, c t r l}}{\delta \tau} \cong \frac{\delta r_{s: f, P D 1}}{\delta \tau}
$$

$\delta \tau=100 \mu$ s (sampling rate limit).

The derivatives were plotted in logarithmic scale as $\frac{\delta \log _{10}\left(r_{s: f}\right)}{\log 10 \cdot r_{s: f} \cdot \delta \tau}$, to facilitate peak identification (not shown).

Oocyte Electrophysiology

The solutions used for the oocyte electrophysiology experiments were prepared as follows. Oocyte ringer (OR) solution contained (in $\mathrm{mM}$ ): $\mathrm{NaCl} 82.5 ; \mathrm{KCl} 2.5 ; \mathrm{CaCl}_{2} 2.5 ; \mathrm{MgCl}_{2} 1 ; \mathrm{HEPES} 5$, adjusted to $\mathrm{pH}$ 7.4 with $\mathrm{NaOH}$. Barth's solution contained (in $\mathrm{mM}$ ): $\mathrm{NaCl}, 88, \mathrm{KCl}, 1, \mathrm{NaHCO}_{3}, 2.4, \mathrm{HEPES}, 10, \mathrm{MgSO}_{4}$, $0.82, \mathrm{Ca}\left(\mathrm{NO}_{3}\right)_{2}, 0.33, \mathrm{CaCl}_{2} 0.41$.

GABA, AMPA and cyclothiazide (CTZ) were purchased from Tocris (Bristol, UK). Salts for experimental solutions were purchased from Sigma-Aldrich (Merck Life Sciences, Milano, Italy).

Human hippocampal membranes were extracted from 3 patients who underwent surgery for drugresistant temporal lobe epilepsy (TLE). The preparation of membranes and the injection procedures have been previously described $[17,18]$. Twelve to 48 hours after membrane injection, the oocytes were placed in a recording chamber (volume, $0.1 \mathrm{ml}$ ) and perfused continuously, $9-10 \mathrm{ml} / \mathrm{min}$, with 
OR at room temperature $\left(20-22^{\circ} \mathrm{C}\right)$. The membrane currents were recorded from voltage clamped oocytes by using two microelectrodes filled with $3 \mathrm{M} \mathrm{KCl}$ [17]. GABA was applied at final concentration of $500 \mu \mathrm{M}$ for $4 \mathrm{~s}$, AMPA was applied at $25 \mu \mathrm{M}$ for $10 \mathrm{~s}$, while oocytes were pretreated for $20 \mathrm{~s}$ with CTZ $(25 \mu \mathrm{M})$. Neurotransmitters were freshly dissolved for each experiment.

PD1 $1_{n-3 D P A}$ was applied to the incubation chamber for 5 minutes to 3 hours or PD $1_{n-3 D P A}$ was directly pressure-injected into the oocytes (intracellular injection in filtered glycine $5 \mathrm{mM}$ ) with a glass micropipette [18].

\section{GABA receptor Immunohistochemistry}

Four male C57BL/6 mice, 11 to 16 week-old were used. Mice were decapitated and their brain removed from the skull. The brain was immediately immersed into ice-cold oxygenated aCSF ( $95 \%$ $\mathrm{O}_{2} / 5 \% \mathrm{CO}_{2}$ ), $\mathrm{pH}=7.2$. Brains were cut upside down in a Vibroslicer (Leica Microsystems VT1000S). From each mouse five to six horizontal $400 \mu \mathrm{m}$ sections of the ventral hippocampus were obtained. The sections were separated by hemisphere and about six sections from the left and six sections from the right hemisphere for each mouse brain were placed into individual wells. Three representative sections of the ventral hippocampus were selected from each hemisphere.

The slices were transferred to a 12 -well plate, each well containing $225 \mu \mathrm{L}$ freshly prepared PD $1_{n-3 D P A}$ solution or vehicle in oxygenated aCSF (3 sections/condition). The sections were incubated at room temperature for $60 \mathrm{~min}$, then transferred to 12 -well plates containing PBS for $15 \mathrm{~min}$, and to $4 \%$ paraformaldehyde (PFA) for 16 hours. Finally, the sections were rinsed twice with PBS for 15 min and subsequently immersed into $20 \%$ sucrose for two subsequent 30 min epochs. Sections were kept in sucrose at $4-6^{\circ} \mathrm{C}$ for 3 days, then cut in a cryostat.

From each of $400 \mu \mathrm{m}$-vibratome sections, three to five anatomically intact $40 \mu \mathrm{m}$ microtome slices were kept in PBS containing $0.01 \%$ sodium azide at $4^{\circ} \mathrm{C}$ and used for immunofluorescence. 
Immunofluorescence analysis

In brief, two slices from each hippocampal section were incubated free floating with $10 \%$ normal goat or horse serum (Biomedica) in Tris-HCl-buffered saline (TBS; $50 \mathrm{~mm}$ ), pH 7.2, containing $0.4 \%$ Triton X-100 (TBS-Triton) for $90 \mathrm{~min}$, followed by incubation with the respective of a rabbit antibody raised against a 7His-fusion protein of amino acids 316-352 of the $\gamma 2$ subunit of the rat GABA receptor protein with maltose binding protein (final concentration $1 \mu \mathrm{g} / \mathrm{ml}$, [19] at room temperature for $16 \mathrm{~h}$, followed by washing with TBS-Triton. Then, slices were incubated with HRP-coupled donkey antirabbit secondary antibody (1:500; catalog \#711035152, Jackson ImmunoResearch; RRID:AB_10015282) at room temperature for $120 \mathrm{~min}$ and then reacted with TSA-Cy3 (homemade; Lumiprobe; $1: 100$ in $50 \mathrm{~mm}$ PBS and $0.005 \% \mathrm{H}_{2} \mathrm{O}_{2}$ ) at room temperature for $5 \mathrm{~min}$. Sections were mounted on slides and covered using Vectashield mounting medium (Vector laboratories, Inc., Burlingame, USA).

The sections were analysed using a fluorescence microscope, and black and white images of the hippocampus were taken at $25 \mathrm{X}$ magnification. The images were evaluated with the Fij//mageJ, program [20]. Using the same circular area, we determined gray values of the strata oriens and radiatum CA1 and CA3, the stratum lacunosum moleculare CA1 and the dentate molecular layer. As background level, we used gray value in the dentate gyrus. Gray values were converted to relative optic densities (ROD) and the background was then subtracted.

\section{Statistics}

Results are presented as mean \pm standard error of mean. Electrophysiology data are depicted in barwhisker plots as the median, range and individual data points of average values per cell, unless otherwise indicated.

Mann-Whitney U test was used to compare groups, and the Kolmogorov-Smirnov test for Empirical Cumulative Distribution comparisons. The differences were considered as statistically significant with $p$ value $<0.01$, and $D$ value $>0.05$. Fisher's exact test was used for analysis of proportion changes. 
For immunohistochemical data, ROD values represent mean (minus background) of four mice, using the average of three $400 \mu \mathrm{m}$ vibratome sections, each estimated as the mean of two $40 \mu \mathrm{m}$ cryotome sections. Statistics was done by Student's t-test. Differences between groups were considered significant for values of $p<0.05$. 


\section{Results}

PD1 $1_{n-3 D P A}$ enhances inhibitory synaptic inputs to CA1 pyramidal neurons

Incubation with PD1 $1_{n-3 D P A}$ increased inhibitory drive onto pyramidal neurons as revealed by decreased interevent interval (IEI) of sIPSCs in cumulative probability curves (Figure 2A) and increased cell-based average frequency of sIPSCs (Figure 2A insert, Table 1). The mIPSCs however showed decreased IEI but no change in cell-based average frequency (Figure $2 \mathrm{~B}$ insert). The amplitude of sIPSCs and mIPSCs showed an increase after PD1 $1_{\text {-3DPA }}$ exposure only when analysed by cumulative probability distributions but failed to demonstrate significant differences when cell-based mean values were compared (Figure 2C, D inserts). Representative traces of spontaneous (s)- and miniature (m)IPSCs are presented in Figure 2G). These data indicate that the most pronounced changes occurred in sIPSC frequencies, as confirmed by both distribution and cell-average analysis, while other changes were only apparent by distribution analysis and not by cell-based averages. Thus, we conclude that PD1 $n$ 3DPA exposure affected predominantly the frequency, and to a lesser extent the amplitude of the sIPSCs. Taken together, these data would support that most of the PD1 $1_{n-3 D P A}$-induced changes occurred in the presynaptic site of inhibitory synapses [21].

Similar analysis as for IPSCs was also applied to excitatory post-synaptic currents (EPSCs) (Figure 3). The results showed no statistically significant changes neither for cumulative probability curves (both for sEPSCs, nor for mEPSCs, respectively), nor for cell-based averaged comparisons of IEI (Figure 3A, B). Similarly, there was no significant effect on sEPSC amplitudes (Figure 3C). There was a minor $(D=0.089)$ increase in mEPSC amplitudes according to Kolmogorov-Smirnov test (with $D=0.089$ ) which wasn't confirmed by cell-based average analysis (Figure 3D). Also sEPSC kinetics were unchanged (Figure 3E), while some minor changes in distributions were detected for mEPSCs in KolmogorovSmirnov test $(D=0.062)$. Overall, incubation of hippocampal slices with PD1 ${ }_{n-3 D P A}$ had no effect on 
sEPSCs and minor, if any effect, on mEPSCs. These minor changes in mEPSCs were negligible as compared to those for IPSCs (see Table 2).

To address the question whether the PD1 $1_{n-3 D P A}$-induced changes in inhibitory transmission were uniformly distributed among peri-somatic and dendritic inhibitory synapses on the pyramidal neurons, we analysed IPSC kinetics. The cumulative probability curves of IPSC rise-times revealed two inflections of the curves (Figure 2E, arrows) around 1 and $3 \mathrm{~ms}$. This suggests that there were two populations of IPSCs with different rise-time kinetics: "fast" and "slow", respectively (Figure 2H). These IPSCs were interpreted as representing peri-somatic (fast) or dendritic (slow) synapse activity, the latter being a result of a passive filtering because of more remote location from the recording patch pipette attached to the cell soma. Interestingly, we observed an increase in rise times for mIPSCs after PD1 $1_{n-3 D P A}$ incubation (Figure $2 F$ ), suggesting that PD1 ${ }_{n-3 D P A}$ might preferentially affect peri-somatic synapses.

In principle, perisomatic IPSCs with fast kinetics should also display larger amplitudes. Indeed, when we plotted IPSC amplitudes against rise times, the high amplitude and fast rise time events had higher amplitude than those with slow kinetics (Figure 4 A, B). Notably, IPSCs with faster kinetics and higher amplitudes displayed a larger increase in amplitudes after PD $1_{n-3 D P A}$ exposure compared to those with slow kinetics and low amplitudes (Figure 4 A, B, magenta). However, only the increase in mIPSCs was statistically significant (see Table 3 for details). This would support that preferentially perisomatic inhibitory synapses onto the CA1 pyramidal neurons were affected by PD1 $1_{n-3 D P A}$ incubation.

\section{$G A B A_{A}$ receptor immunohistochemistry}

Although the results of electrophysiological experiments pointed towards a preferential presynaptic mode of PD1 $1_{n-3 D P A}$ action, cumulative probability curves suggested a potential effect on IPSC amplitudes, therefore postsynaptic changes (e. g., in $\mathrm{GABA}_{\mathrm{A}}$ receptors) could also be present. Consequently, we investigated whether PD1 $1_{n-3 D P A}$ could have induced changes in postsynaptic $\mathrm{GABA}_{\mathrm{A}}$ receptor subunit composition. $\mathrm{GABA}_{\mathrm{A}}$ receptors are the major inhibitory receptors on principal 
neurons and represent chloride channels assembled by five heterogeneous subunits. These subunits derive from about 15 different genes. A majority of $\mathrm{GABA}_{A}$ receptors contain a $\gamma$-subunit [19]. Together with a variety of scaffold proteins, like gephyrin, this subunit appears to contribute to the assembly of the receptor complex $[22,23]$. We therefore incubated hippocampal slices with PD1 $1_{n-3 D P A}$ and by immunofluorescence assessed the expression of the $\mathrm{GABA}_{A}$ receptor $\gamma$-subunit in six different subfields (Figure 5) of the hippocampus (Table 4).

Following PD1 $1_{n-3 D P A}$ incubation, minor fluorescence differences were observed in all hippocampal fields, compared to control slices, and these alterations were not statistically significant (Table 4). The representative slices with immunoflourescence stainig in gray-scale are presented on Figure 5 . The data therefore did not support major changes in $\mathrm{GABA}_{\mathrm{A}}$ receptor $\gamma$-subunit composition, indirectly reinforcing preferetially presynaptic effect of PD $1_{n-3 D P A}$.

\section{Oocyte electrophysiology}

To further strengthen the immunostaining results, we performed oocyte electrophysiology since the oocyte membranes incorporate exogenous neuronal membranes fragments containing endogenously expressed GABA and glutamate receptors (Palma et al., 2002, Ruffolo et al., 2018). We examined GABA and AMPA currents in oocytes incubated with PD1 $1_{n-3 D P A}$ or after intra-oocyte injection of PD1 $1_{n-}$ 3DPA to test if there was an intracellular site of PD1 $1_{n-3 D P A}$ interaction with the membrane receptors. No significant changes either in GABA or AMPA currents were observed in these experiments at any timepoint of incubation (Table 5). These data support the slice electrophysiology results that PD1 $n$ 3DPA-induced changes in IPSCs are generated at presynaptic sites and that PD $1_{n-3 D P A}$ does not alter postsynaptic $\mathrm{GABA}_{\mathrm{A}}$ receptors. Moreover, PD1 $1_{\mathrm{n}-3 \mathrm{DPA}}$ appears ineffective on glutamatergic excitatory synaptic transmission.

\section{PD1 ${ }_{n-3 D P A}$ effect is blocked by Pertussis toxin}

Next, we hypothesised that PD1 $1_{n-3 D P A}$ effect could be mediated via some type of GPCRs. Since, to our best knowledge, there are no data available on which type of GPCR could be involved in PD1 $1_{n-3 D P A}$ 
signalling, we used pertussis toxin, a non-specific GPCR antagonist, to address this question. Pertussis toxin $(1 \mu \mathrm{g} / \mathrm{mL})$ incubation reversed the increase in frequency (both cumulative probability curves and cell-based average frequency), amplitude and kinetics (only cumulative probability curves) of spontaneous IPSC induced by PD1 $1_{\text {-3DPA }}$ (Figure 6) (K-S $p<0.001$ for distribution changes relative to PD1 $1_{n-3 D P A}$ incubation, cell-averaged comparison statistically significant for the frequency reversal Table 6). These data suggest that PD $1_{\mathrm{n}-3 \mathrm{DPA}}$ increase inhibitory drive onto the pyramidal neurons by interaction with a GPCR. Further studies are required however to identify the specific type of the GPCR that is responsible for this effect.

\section{Discussion}

Here we demonstrate that PD1 $1_{\text {-3DPA }}$ applied to hippocampal slices enhances inhibitory drive onto the CA1 pyramidal neurons. Interestingly, most of this increase was observed in peri-somatic inhibitory synapses. No changes were observed in excitatory synapses onto the pyramidal neurons. In addition, we demonstrate that this effect of PD1 $1_{n-3 D P A}$ is mediated by a yet unidentified GPCR.

Our findings provide fresh evidence that, in addition to its previously reported pro-resolving and antiinflammatory actions, PD1 $1_{n-3 D P A}$ mediates a direct effect on network excitability, by interacting with GPCR. This newly described mechanism of action of PD1 $1_{n-3 D P A}$ may contribute to the anti-seizure and neuroprotective effects described in vivo [11]. Notably, PD1 $1_{n-3 D P A}$ was among the most abundant lipid mediators measured during epilepsy development in mice suggesting it might be a key player in the tissue attempt to engage resolution pathways. Accordingly, early post-injury administration of PD1 $n$ 3DPA significantly reduced neuroinflammation, in line with previous findings that PD $1_{n-3}$ DPA and its precursor n-3 DPA [24] exert powerful antiinflammatory effects [25]. This anti-inflammatory effect was associated with a drastic reduction in the number and duration of spontaneous seizures in mice. The $10 \mathrm{nM}$ concentration we used in hippocampal slices was previously shown to mediate antiinflammatory effects of the molecule in vitro [26]. When PD1 $1_{\text {-3DPA }}$ was applied in vivo (200 ng, 552.5 
$\mu \mathrm{M}$ injected concentration) [11] into the ventricle, the original concentration was diluted in the CSF, which in mice is approximately $35 \mu \mathrm{L}$ [27], resulting in a final concentration of $\sim 15.8 \mu \mathrm{M}$. However, PD1 $1_{\text {-3DPA }}$ had to diffuse to the hippocampal tissue, along a concentration gradient defined by the distance to the target tissue, diffusion coefficient etc, resulting in further dilution. Therefore, it is difficult to compare the in vivo concentration effective on seizures with the in vitro concentration affecting GABA neurotransmission. However, the concentration we applied to the slices has been defined as optimal by the previous in vitro reports [26], and appears to be sufficient to elicit an effect. Therefore, the mechanism of action of PD $1_{n-3 D P A}$ identified by the present in vitro study is likely to be relevant in vivo when PD1 $1_{n-3 D P A}$ is injected into the ventricles and suppress seizures. Thus, increased inhibitory drive on pyramidal neurons of hippocampus caused by direct application of PD $1_{n-3 D P A}$ may counteract increased network excitability and thereby reduce spontaneous recurrent seizures.

An interesting finding of this study is that the effect of PD1 $1_{n-3 D P A}$ was predominantly exerted on perisomatic inhibitory synapses. Although our data on cumulative probability curves demonstrating increased amplitude of IPSCS may indicate also a postsynaptic action of PD1 $1_{n-3 D P A}$ on inhibitory synapses, both immunohistochemical analysis of postsynaptic $\mathrm{GABA}_{A}$ receptors and oocyte assay failed to demonstrate changes at postsynaptic sites. The oocyte assay has been proven to be effective in identifying alteration in $\mathrm{GABA}_{\mathrm{A}}$ receptor function [28]. One could speculate that PD1 $1_{\mathrm{n}-3 \mathrm{DPA}}$ acts mostly presynaptically on CA1 pyramidal neurons, and selectively affects those inhibitory interneurons that innervate perisomatic area of the principal neurons, such as, for example, parvalbumin-expressing interneurons (PV-interneurons) $[29,30]$. Since we show that the PD $1_{n-3 D P A}$ effect is sensitive to pertussis toxin, it implies that PV-interneurons may selectively express a specific type of GPCRs and/or associated $G_{o} / G_{i}$ proteins that mediate the PD1 $1_{n-3 D P A}$ effect. This hypothesis is worth of further investigation. 
In view of its neuromodulatory effects, PD1 $1_{n-3 D P A}$ may prove to be an attractive target for anti-ictogenic and anti-epileptileptogenic interventions. In this regard, identifying a specific GCPR that mediates its effect would be of particular interest.

Our data raise the possibility that PD1 $1_{n-3 D P A}$ might also counteract in vivo the effects of ictogenic cytokines such as IL-1 $\beta$ and TNF which are proven to decrease inhibitory synaptic transmission (see [5]).

In conclusion, we have identified a novel mechanism by which a key pro-resolving molecule affects network excitability, in addition to its antiinfammatory effects. This direct neuronal mechanism involves yet unidentified a GPCR, elucidation of which may provide a novel target for antiepileptogenic and anti-ictogenic approaches in epilepsy.

\section{Acknowledgements.}

The authors would like to thank Prof Trond Vidar Hansen (University of Oslo) for providing PD $1_{n-3}$ DPA. GS would like to thank Anna Wieselthaler-Hoelzl for technical help.

Consent to participate and Consent for publication.

\section{References}

1. Dawit S, Crepeau AZ (2020) When Drugs Do Not Work: Alternatives to Antiseizure Medications. Curr Neurol Neurosci Rep 20:37. https://doi.org/10.1007/s11910-020-01061-3

2. Cascino GD, Brinkmann BH (2021) Advances in the Surgical Management of Epilepsy: DrugResistant Focal Epilepsy in the Adult Patient. Neurologic Clinics 39:181-196. https://doi.org/10.1016/j.ncl.2020.09.010

3. Vezzani A, Balosso S, Ravizza T (2019) Neuroinflammatory pathways as treatment targets and biomarkers in epilepsy. Nat Rev Neurol 15:459-472. https://doi.org/10.1038/s41582-0190217-x

4. Vliet EA van, Aronica E, Vezzani A, Ravizza T (2018) Review: Neuroinflammatory pathways as treatment targets and biomarker candidates in epilepsy: emerging evidence from preclinical and clinical studies. Neuropathology and Applied Neurobiology 44:91-111. https://doi.org/10.1111/nan.12444

5. Vezzani A, Viviani B (2015) Neuromodulatory properties of inflammatory cytokines and their impact on neuronal excitability. Neuropharmacology 96:70-82.

https://doi.org/10.1016/j.neuropharm.2014.10.027 
6. Frigerio F, Pasqualini G, Craparotta I, et al (2018) n-3 Docosapentaenoic acid-derived protectin D1 promotes resolution of neuroinflammation and arrests epileptogenesis. Brain 141:31303143. https://doi.org/10.1093/brain/awy247

7. Weissberg I, Wood L, Kamintsky L, et al (2015) Albumin induces excitatory synaptogenesis through astrocytic TGF- $\beta$ /ALK5 signaling in a model of acquired epilepsy following blood-brain barrier dysfunction. Neurobiol Dis 78:115-125. https://doi.org/10.1016/j.nbd.2015.02.029

8. Devinsky O, Vezzani A, Najjar S, et al (2013) Glia and epilepsy: excitability and inflammation. Trends in Neurosciences 36:174-184. https://doi.org/10.1016/j.tins.2012.11.008

9. Roseti C, van Vliet EA, Cifelli P, et al (2015) GABAA currents are decreased by IL-1 $\beta$ in epileptogenic tissue of patients with temporal lobe epilepsy: implications for ictogenesis. Neurobiology of Disease 82:311-320. https://doi.org/10.1016/j.nbd.2015.07.003

10. Serhan CN, Chiang N, Dalli J (2018) New pro-resolving n-3 mediators bridge resolution of infectious inflammation to tissue regeneration. Molecular Aspects of Medicine 64:1-17. https://doi.org/10.1016/j.mam.2017.08.002

11. Frigerio F, Pasqualini G, Craparotta I, et al (2018) n-3 Docosapentaenoic acid-derived protectin D1 promotes resolution of neuroinflammation and arrests epileptogenesis. Brain 141:31303143. https://doi.org/10.1093/brain/awy247

12. Xiao Y-F, Li X (1999) Polyunsaturated fatty acids modify mouse hippocampal neuronal excitability during excitotoxic or convulsant stimulation. Brain Research 846:112-121. https://doi.org/10.1016/S0006-8993(99)01997-6

13. Taha AY, Zahid T, Epps T, et al (2013) Selective reduction of excitatory hippocampal sharp waves by docosahexaenoic acid and its methyl ester analog ex-vivo. Brain Research 1537:9-17. https://doi.org/10.1016/j.brainres.2013.09.004

14. Lein ES, Hawrylycz MJ, Ao N, et al (2007) Genome-wide atlas of gene expression in the adult mouse brain. Nature 445:168-176. https://doi.org/10.1038/nature05453

15. Jonas P, Major G, Sakmann B (1993) Quantal components of unitary EPSCs at the mossy fibre synapse on CA3 pyramidal cells of rat hippocampus. J Physiol 472:615-663

16. Mikroulis A (2021) xPSC-detection - Template correlation-based detection of postsynaptic currents.

17. Palma E, Esposito V, Mileo AM, et al (2002) Expression of human epileptic temporal lobe neurotransmitter receptors in Xenopus oocytes: An innovative approach to study epilepsy. Proc Natl Acad Sci U S A 99:15078-15083. https://doi.org/10.1073/pnas.232574499

18. Conti L, Palma E, Roseti C, et al (2011) Anomalous levels of Cl-transporters cause a decrease of GABAergic inhibition in human peritumoral epileptic cortex. Epilepsia 52:1635-1644. https://doi.org/10.1111/j.1528-1167.2011.03111.x

19. Pirker S, Schwarzer C, Wieselthaler A, et al (2000) GABAA receptors: immunocytochemical distribution of 13 subunits in the adult rat brain. Neuroscience 101:815-850. https://doi.org/10.1016/S0306-4522(00)00442-5 
20. Schindelin J, Arganda-Carreras I, Frise E, et al (2012) Fiji - an Open Source platform for biological image analysis. Nat Methods 9:. https://doi.org/10.1038/nmeth.2019

21. Markram H, Tsodyks M (1996) Redistribution of synaptic efficacy between neocortical pyramidal neurons. Nature 382:807-810. https://doi.org/10.1038/382807a0

22. Essrich C, Lorez M, Benson JA, et al (1998) Postsynaptic clustering of major GABA A receptor subtypes requires the $\gamma 2$ subunit and gephyrin. Nature Neuroscience 1:563-571. https://doi.org/10.1038/2798

23. Khayenko V, Maric HM (2019) Targeting GABAAR-Associated Proteins: New Modulators, Labels and Concepts. Front Mol Neurosci 12:. https://doi.org/10.3389/fnmol.2019.00162

24. Ferdinandusse S, Denis S, Mooijer PA, et al (2001) Identification of the peroxisomal betaoxidation enzymes involved in the biosynthesis of docosahexaenoic acid. J Lipid Res 42:19871995

25. Dalli J, Colas RA, Serhan CN (2013) Novel n-3 immunoresolvents: structures and actions. Sci Rep 3:1940. https://doi.org/10.1038/srep01940

26. Gobbetti T, Dalli J, Colas RA, et al (2017) Protectin D1n-3 DPA and resolvin D5n-3 DPA are effectors of intestinal protection. PNAS 114:3963-3968.

https://doi.org/10.1073/pnas.1617290114

27. Pardridge WM (2016) CSF, blood-brain barrier, and brain drug delivery. Expert Opinion on Drug Delivery 13:963-975. https://doi.org/10.1517/17425247.2016.1171315

28. Ruffolo G, Cifelli P, Roseti C, et al (2018) A novel GABAergic dysfunction in human Dravet syndrome. Epilepsia 59:2106-2117. https://doi.org/10.1111/epi.14574

29. Jinno S, Klausberger T, Marton LF, et al (2007) Neuronal Diversity in GABAergic Long-Range Projections from the Hippocampus. J Neurosci 27:8790-8804.

https://doi.org/10.1523/JNEUROSCI.1847-07.2007

30. Klausberger T, Somogyi P (2008) Neuronal Diversity and Temporal Dynamics: The Unity of Hippocampal Circuit Operations. Science 321:53-57. https://doi.org/10.1126/science.1149381

\section{Tables}

Table 1. Frequency and amplitude comparison of cell-averaged IPSCs between control and PD1 $1_{n-3 D P A}$ treated slices.

\begin{tabular}{|l|l|l|l|l|}
\hline \multirow{2}{*}{} & \multicolumn{2}{|l|}{ frequency (1/s) } & \multicolumn{2}{l|}{ amplitude (pA) } \\
\cline { 2 - 5 } & sIPSCs & mIPSCs & sIPSCs & mIPSCs \\
\hline Vehicle & $3.717 \pm 0.536, \mathrm{n}=$ & $3.949 \pm 0.695, \mathrm{n}$ & $11.266 \pm 2.048, \mathrm{n}$ & $13.804 \pm 4.926, \mathrm{n}=$ \\
& 8 & $=5$ & $=8$ & 5 \\
\hline
\end{tabular}




\begin{tabular}{|l|l|l|l|l|}
\hline PD1 ${ }_{n-3 D P A}$ & $5.143 \pm 0.476, \mathrm{n}=$ & $5.048 \pm 0.595, \mathrm{n}$ & $20.98 \pm 4.541, \mathrm{n}=$ & $19.133 \pm 4.263, \mathrm{n}=$ \\
& 9 & $=8$ & 9 & 8 \\
\hline Mann Whitney U & 0.046 & 0.171 & 0.068 & 0.136 \\
test $\mathrm{p}$ & & & & \\
\hline
\end{tabular}

Table 2.Frequency and amplitude comparison of cell-averaged EPSCs between control and PD $1_{n-3 D P A}$ treated slices.

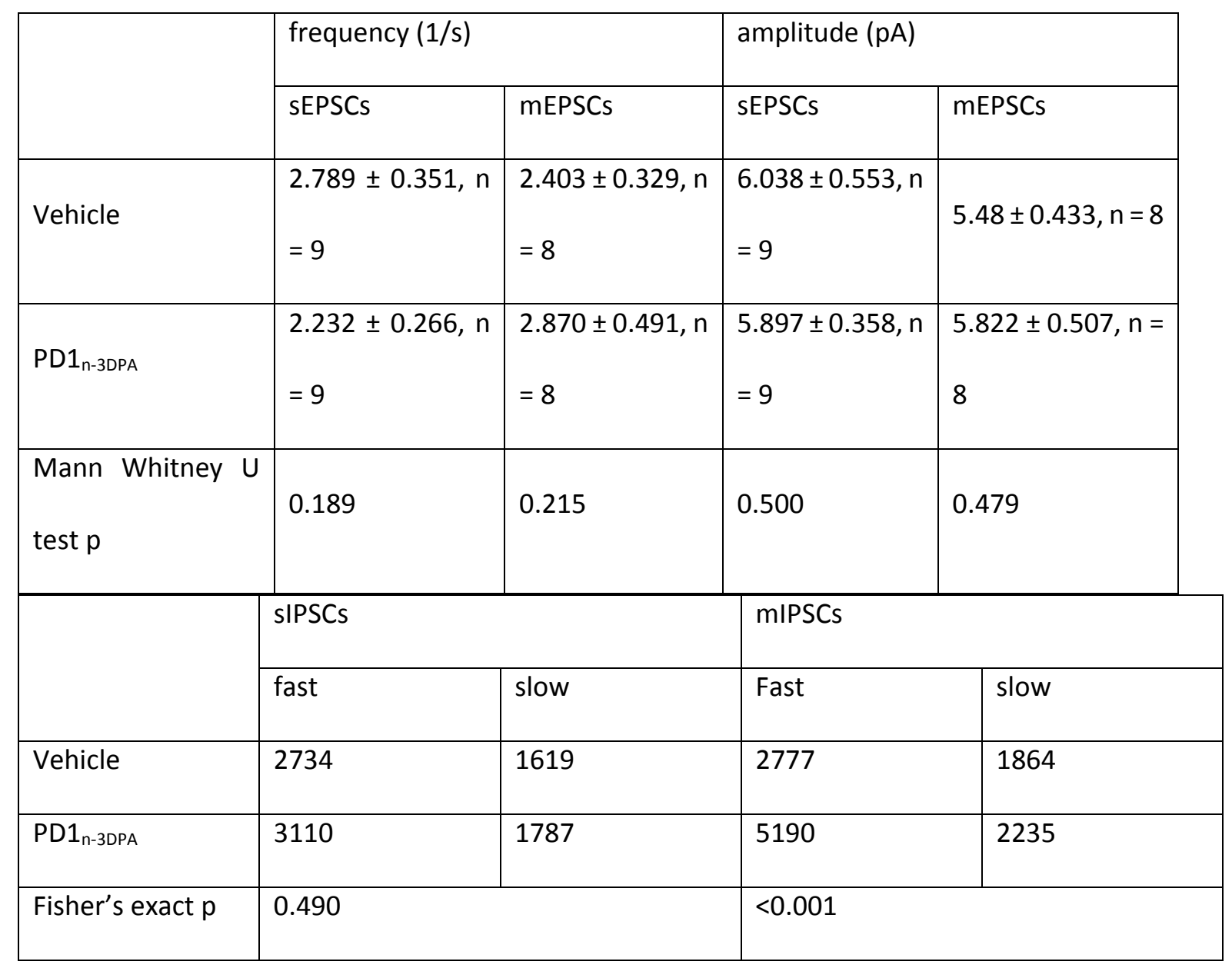

Table 3. Increased proportion of fast rise-time IPSC counts following PD1 $1_{n-3 D P A}$ incubation. Rise times separated at $1.4 \mathrm{~ms}$.

Table 4. Data represent the mean \pm sem of values from 4 vibratome slices (incubated with aCSF or $\left.P D 1_{n-3 D P A}\right)$ each corresponding to an individual mouse. Each data point is based on average ROD 
values from five to six $40 \mu \mathrm{m}$ sections/vibratome slice. DG-ML, molecular layer of the dentate gyrus, so, stratum oriens, sr, stratum radiatum, slm, stratum lacunosum moleculare.

\begin{tabular}{|c|c|c|c|c|c|c|c|c|c|c|c|}
\hline & \multicolumn{2}{|l|}{ DG-ML } & \multicolumn{2}{|l|}{ CA1 so } & \multicolumn{2}{|l|}{ CA1 sr } & \multicolumn{2}{|c|}{ CA1 slm } & \multicolumn{2}{|l|}{ CA3 so } & CA3 sr \\
\hline aCSF & \multicolumn{2}{|c|}{$0.200 \pm 0.019$} & \multicolumn{2}{|c|}{$0.217 \pm 0.038$} & \multicolumn{2}{|c|}{$0.251 \pm 0.037$} & \multicolumn{2}{|c|}{$0.213 \pm 0.024$} & \multicolumn{2}{|c|}{$0.164 \pm 0.019$} & $0.160 \pm 0$ \\
\hline \multirow[t]{2}{*}{$P D 1_{n-3 D P A}$} & $0.229 \pm$ & 0.011 & $0.243 \pm$ & 0.037 & $0.282 \pm$ & 0.007 & $0.215 \pm$ & 0.023 & $0.125 \pm$ & 0.007 & $0.165 \pm$ \\
\hline & $(115 \%)$ & & $(112 \%)$ & & $(112 \%)$ & & (99\%) & & $(76 \%)$ & & $(102 \%)$ \\
\hline$P$-value & 0.236 & & 0.531 & & 0.363 & & 0.973 & & 0.089 & & 0.861 \\
\hline
\end{tabular}

Table 5. Results of PD1 $1_{n-3 D P A}$ incubation and its intracellular injection in oocytes injected with human hippocampal tissue

\begin{tabular}{|c|c|c|c|c|}
\hline Incubation Time & $\begin{array}{l}\text { GABA current } \\
\left.\text { (pre-PD1 } 1_{n-3 D P A}\right)\end{array}$ & $\begin{array}{l}\text { GABA current } \\
\left.\text { (post-PD1 } 1_{n-3 D P A}\right)\end{array}$ & $\begin{array}{l}\text { AMPA current } \\
\left.\text { (pre-PD } 1_{n-3 D P A}\right)\end{array}$ & $\begin{array}{l}\text { AMPA current } \\
\left.\text { (post-PD } 1_{\text {n-3DPA }}\right)\end{array}$ \\
\hline 5 minutes & $\begin{array}{l}87.7 \pm 16.0 \mathrm{nA} \\
(\mathrm{n}=6)\end{array}$ & $\begin{array}{l}88.3 \pm 14.0 \mathrm{nA} \\
(\mathrm{n}=6)\end{array}$ & - & - \\
\hline 20 minutes & $\begin{array}{l}54.9 \pm 23.1 \mathrm{nA} \\
(\mathrm{n}=6)\end{array}$ & $\begin{array}{l}54.4 \pm 18.9 \mathrm{nA} \\
(\mathrm{n}=6)\end{array}$ & -- & -- \\
\hline 1 hour & $\begin{array}{l}75.0 \pm 10 n A \\
(n=7)\end{array}$ & $\begin{array}{l}77.0 \pm 8 n A \\
(n=7)\end{array}$ & $\begin{array}{l}19.3 \pm 1.5 \mathrm{nA} \\
(\mathrm{n}=5)\end{array}$ & $\begin{array}{l}18.3 \pm 1.8 \\
(n=5)\end{array}$ \\
\hline 2 hours & $\begin{array}{l}54.7 \pm 15.7 \mathrm{nA} \\
(\mathrm{n}=18)\end{array}$ & $\begin{array}{l}54.3 \pm 13.9 \mathrm{nA} \\
(n=18)\end{array}$ & $\begin{array}{l}26.9 \pm 3.8 n A \\
(n=6)\end{array}$ & $\begin{array}{l}23.0 \pm 5, .2 \mathrm{nA} \\
(\mathrm{n}=6)\end{array}$ \\
\hline 3 hours & $\begin{array}{l}58.5 \pm 17.0 \mathrm{nA} \\
(\mathrm{n}=17)\end{array}$ & $\begin{array}{l}57.0 \pm 15.5 \mathrm{nA} \\
(\mathrm{n}=17)\end{array}$ & $\begin{array}{l}29.4 \pm 3.5 \mathrm{nA} \\
(n=6)\end{array}$ & $\begin{array}{l}23.0 \pm 7.5 \mathrm{nA} \\
(\mathrm{n}=6)\end{array}$ \\
\hline $\begin{array}{l}\text { Intracellular } \\
\text { injection }\end{array}$ & $\begin{array}{l}70.7 \pm 20.4 \mathrm{nA} \\
(\mathrm{n}=7)\end{array}$ & $\begin{array}{l}64.8 \pm 22.7 \mathrm{nA} \\
(\mathrm{n}=7)\end{array}$ & $\begin{array}{l}7.6 \mathrm{nA} \pm 1.7 \mathrm{nA} \\
(\mathrm{n}=4)\end{array}$ & $\begin{array}{l}6.2 \mathrm{nA} \pm 1.5 \mathrm{nA} \\
(\mathrm{n}=4)\end{array}$ \\
\hline
\end{tabular}


Table 6. Cell-averaged sIPSC frequency and amplitude comparison between PD $1_{n-3 D P A}$ and PD $1_{n-3 D P A}+$ pertussis toxin incubations. $n=545$ events per cell.

\begin{tabular}{|c|c|c|}
\hline & frequency $(1 / \mathrm{s})$ & amplitude (pA) \\
\hline$P D 1_{n-3 D P A}$ & $5.143 \pm 0.476, n=9$ & $20.98 \pm 4.541, n=9$ \\
\hline$P D 1_{n-3 D P A}+$ pertussis toxin & $3.324 \pm 0.408, n=7$ & $16.797 \pm 4.874, n=7$ \\
\hline Mann Whitney $U$ test $p$ & 0.010 & 0.263 \\
\hline
\end{tabular}

\section{Figure captions}

Figure 1. Images illustrating hippocampal slicing. A, B: Approximate slicing location, according to the Allen Mouse Brain Atlas. squares in B indicate the area of the slices kept. Images generated with the Allen Institute Brain Explorer 2 software, using the Allen Mouse Brain Atlas [14] C: example of slice section after electrophysiology (DAPI staining in blue).

Figure 2. PD1 $1_{n-3 D P A}$ incubation increases the frequency and amplitude of IPSCs recorded in CA1 pyramidal neurons. (A) sIPSC interevent interval (IEI) presented as cumulative probability curves (Kolmogorov-Smirnov test $D=0.098, p<0.01$ ) and cell-based averages (insert) of sIPSC frequency. (B) mIPSCs, same presentation as (A), IEI (Kolmogorov-Smirnov test $D=0.102, p<0.01$ ) and average frequencies. (C) and (D) sIPSC and mIPSC amplitudes, respectively, presented as cumulative probability curves (Kolmogorov-Smirnov test sIPSCS: $D=0.272, p<0.01 ;$ mIPSCs: $D=0.181, p<0.01$ ) and cell-based averages (insert). (E) and (F) rise-times of sIPSCs and mIPSCs, respectively, presented as cumulative probability curves (Kolmogorov-Smirnov test sIPSCS: $D=0.029, p>0.01$; mIPSCs: $D=0.101$, $\mathrm{p}<0.01$ ) and cell-based averages (inserts). Arrows denote 2 distinct distribution peaks around 1 and 3 ms. Insets display the median and range of cell averages. * Mann-Whitney $p<0.05$ for cell averages. (G) representative traces of sIPSCs with and without PD $1_{n-3 D P A}$. Highlighted segment shows 10 -fold time-stretched recording. $(\mathrm{H})$ magnification of amplitude-normalised averaged fast (black) and slow (grey) rise-time events in cells from control slices ( $n=125$ sIPSCs per group).

Figure 3. Effect of PD1 $1_{n-3 D P A}$ incubation on EPSCs recorded in CA1 pyramidal neurons. PD1 $1_{n-3 D P A}$ incubation did not significantly affects sEPSCs or mEPSCs. (A) and (B), sEPSCs and mEPSCs, respectively, are presented as cumulative probability curves for IEI (sEPSCs: Kolmogorov-Smirnov test $D=0.014, p<0.01 ;$ mEPSCs: $D=0.045, p<0.01$ ) and cell-based averages for $s E P S C$ frequencies (inserts). (C) and (D) SEPSCS and mEPSCs, respectively, are presented as cumulative probability curves (sEPSCs: Kolmogorov-Smirnov test $D=0.045, p<0.01$; mEPSCs: $D=0.089, p<0.01$ ) for amplitudes and cell-based averages (inserts). (E) and (F) SEPSC and mEPSC rise-times, respectively, are presented as cumulative probability curves (sEPSCs: Kolmogorov-Smirnov test $D=0.028, p>0.01$; mEPSCs: $D=0.062, p<0.01$ ) and cell-based averages. Insets display the median and range of cell average. Arrows denote much less pronounced peaks as compared to those in Figure 1). (G) representative traces with and without PD1 $1_{n-3 D P A}$. Highlighted segment shows 10 -fold time-stretched recording. $(\mathrm{H})$ magnification of amplitude-normalised averaged fast (black) and slow (grey) rise-time events in cells from control slices ( $n=42$ sEPSCs per group).

Figure 4. Rise time - amplitude distribution of IPSCs. Spontaneous (A) and miniature (B) IPSCs, after vehicle (green) and PD1 ${ }_{n-3 D P A}$ incubation (magenta). Fast rise time events with high amplitudes, 
around the $1^{\text {st }}$ distribution peak $(\leq 1.4 \mathrm{~ms})$ were proportionally increased after PD $1_{n-3 D P A}$ incubation. Fisher's exact test ns (sIPSCs), $\mathrm{p}<0.05$ (mIPSCs).

Figure 5. Example of slices and the subfields examined with immunohistochemistry. Example of a control (A-C) and and PD1 ${ }_{n-3 D P A}$-incubated slice (D-F). B, E: CA1 fields, D, F: CA3 fields.

Immunostainings are in white colour.

Figure 6. PD $1_{n-3 D P A}$ effect on IEI of IPSC in CA1 pyramidal neurons was reverted by pertussis toxin. The proportion of fast to slow IPSC rise times was also further reduced. Control (vehicle) group is displayed as a reference. Insets display the median and range of cell averages. * Mann-Whitney $p$ $<0.05$ for cell averages, Kolmogorov-Smirnov $p<0.01, D>0.1$ for all PD $1_{n-3 D P A}-P D 1_{n-3 D P A}+P$ toxin distribution comparisons. 


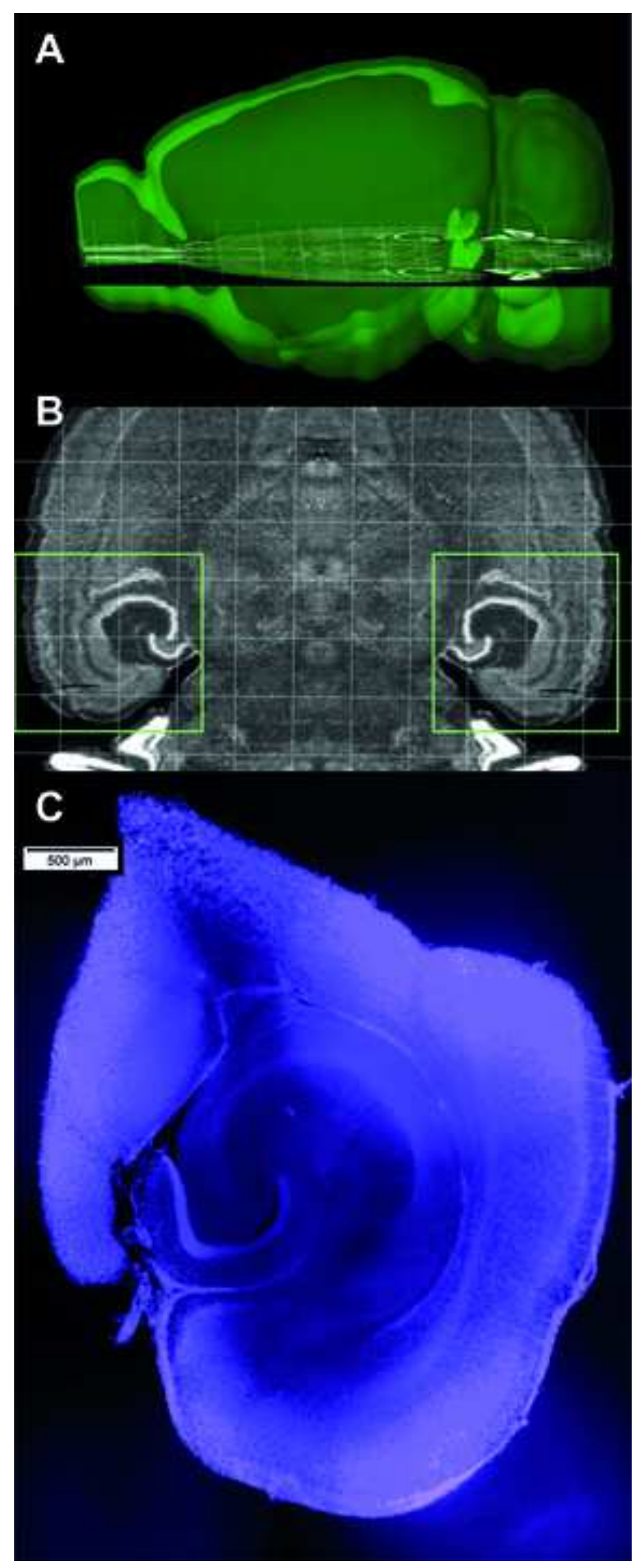




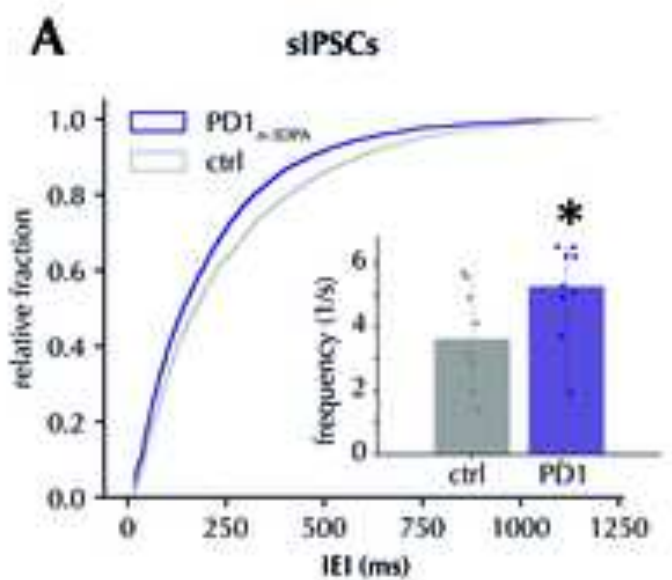

B mipscs

C

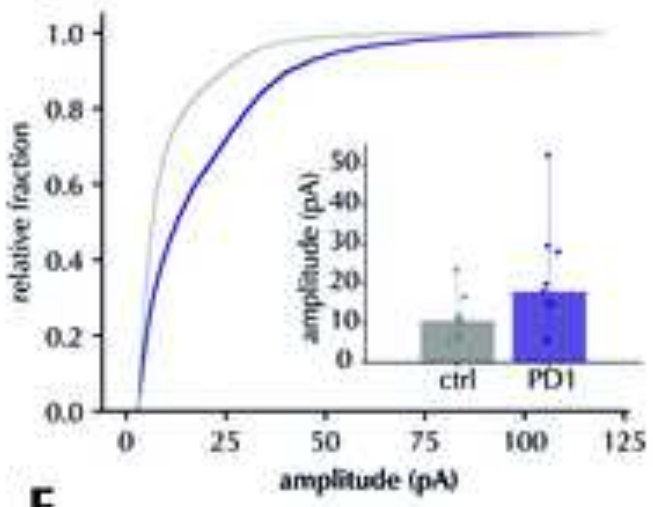

E

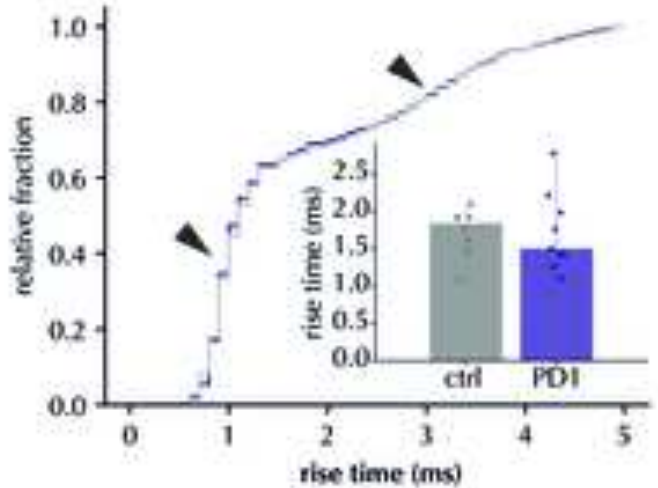

G SIPSCs ictit:

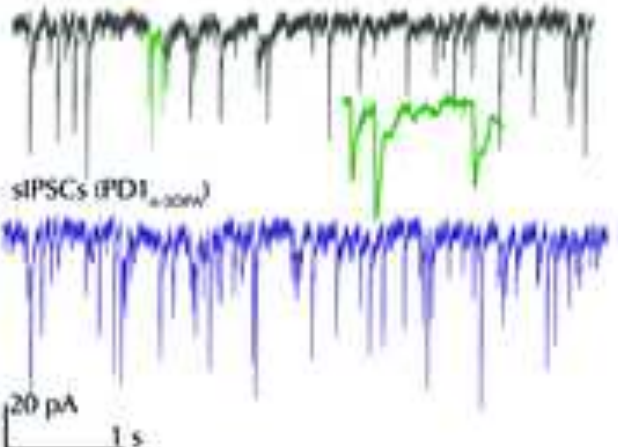

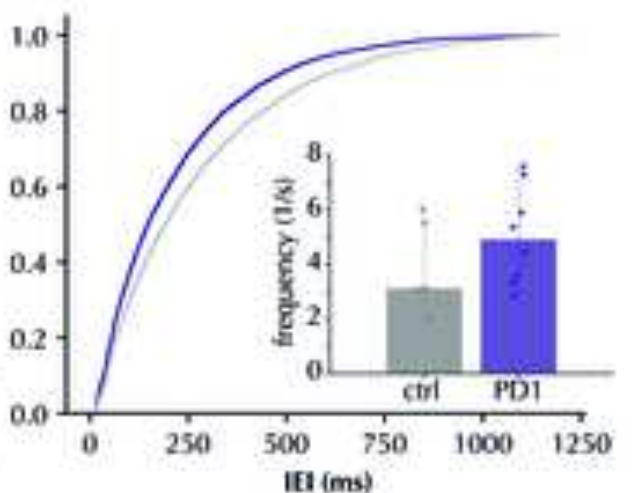

D

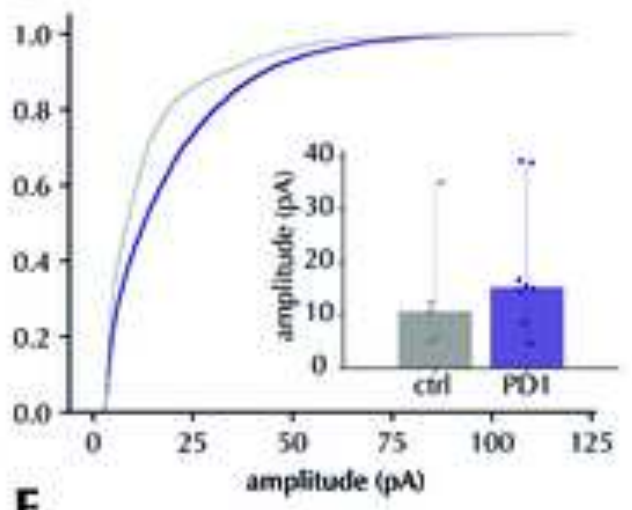

F

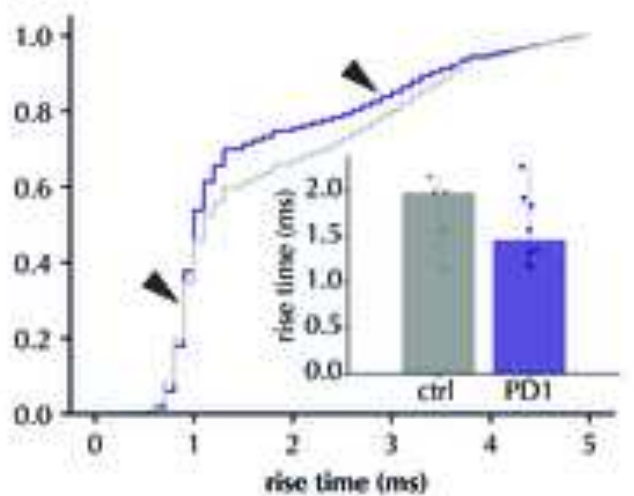

H

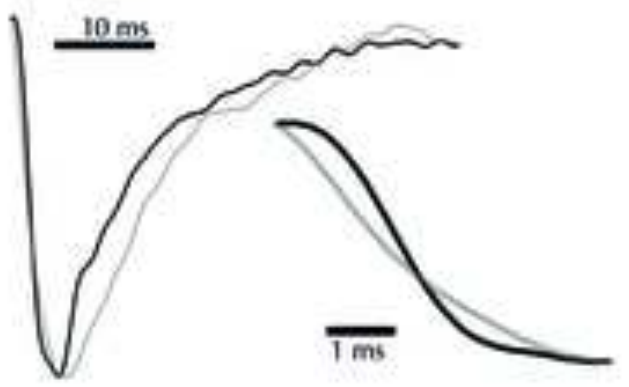


$\begin{array}{llll}\text { A } & \text { SEPSCS } & \text { B } & \text { mEPSCs }\end{array}$
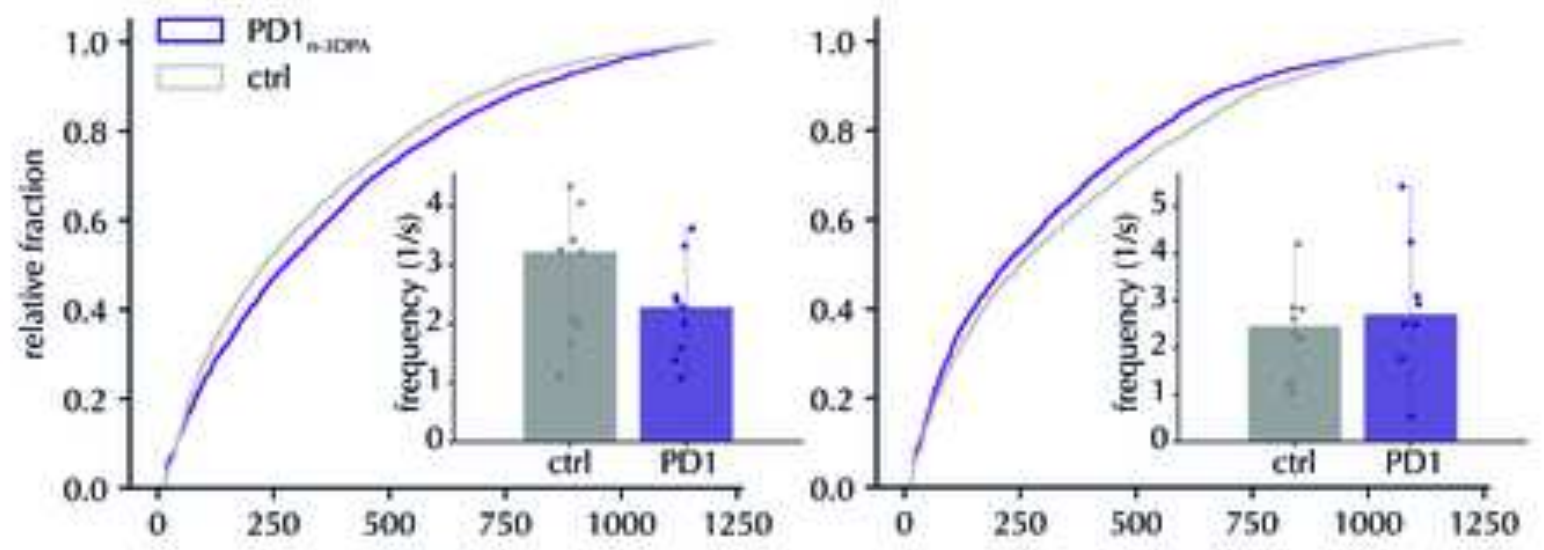
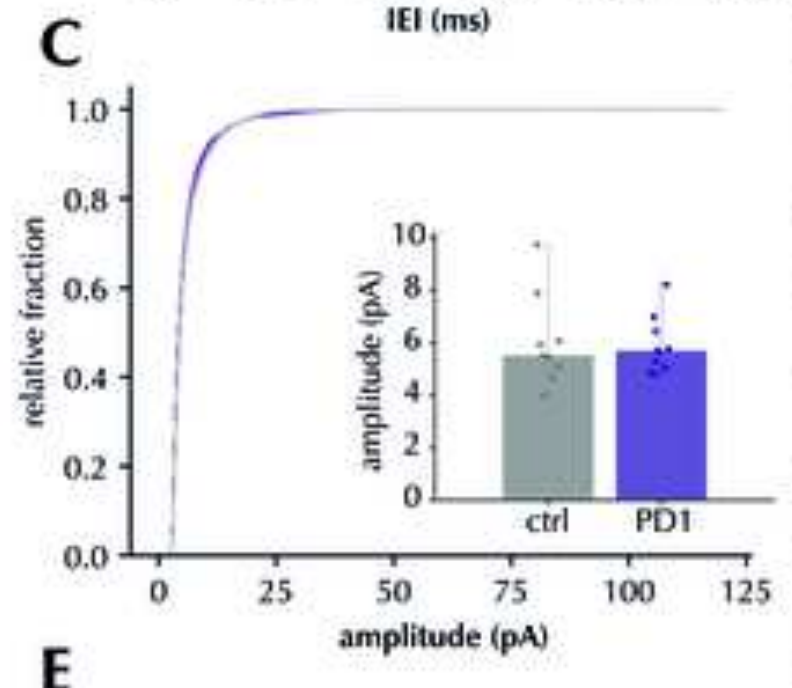

E

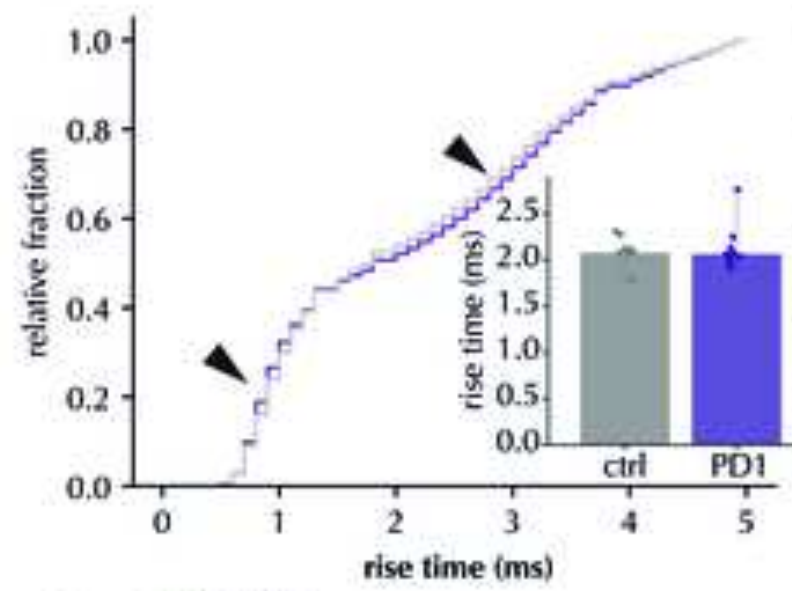

G SEPSCs (ctrl)

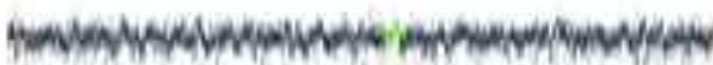

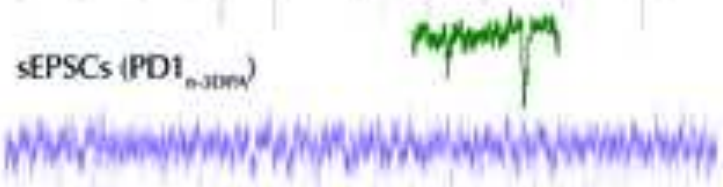

$10 \mathrm{pA}$
D
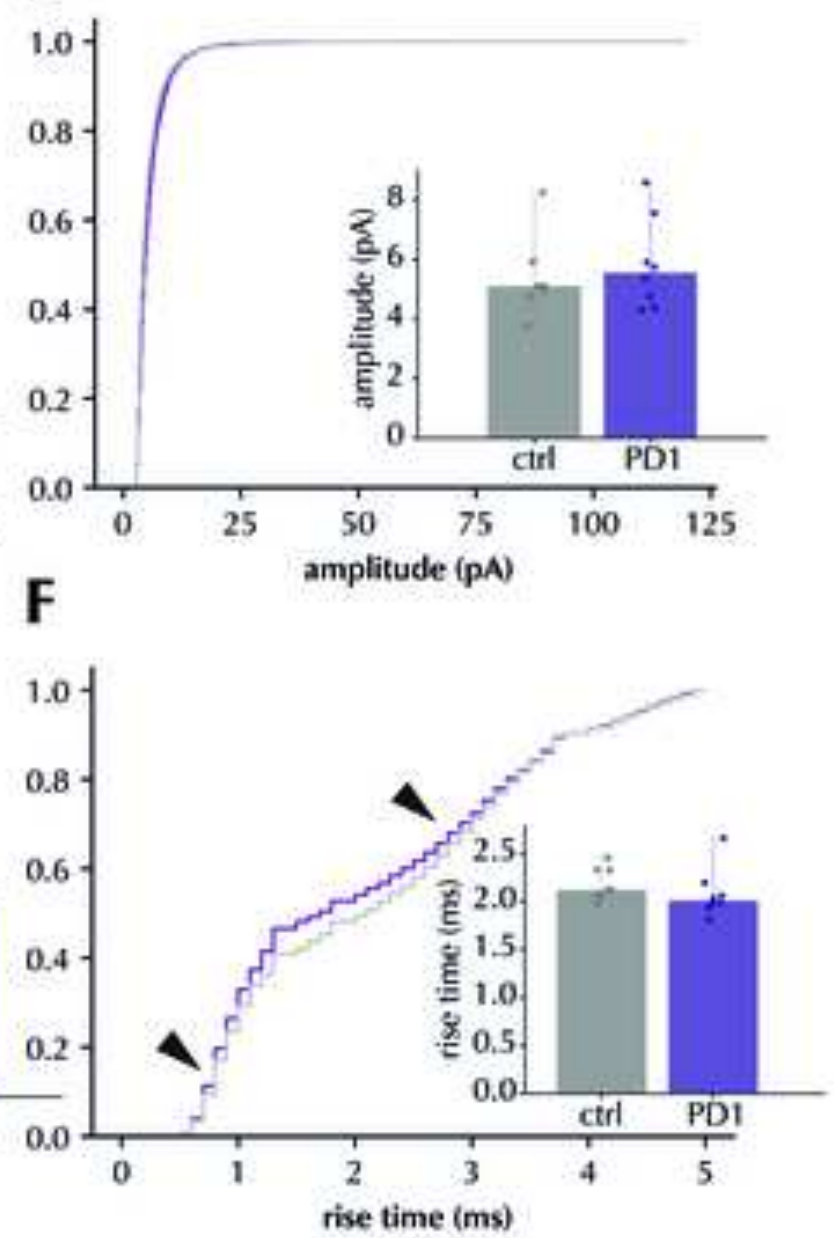

H

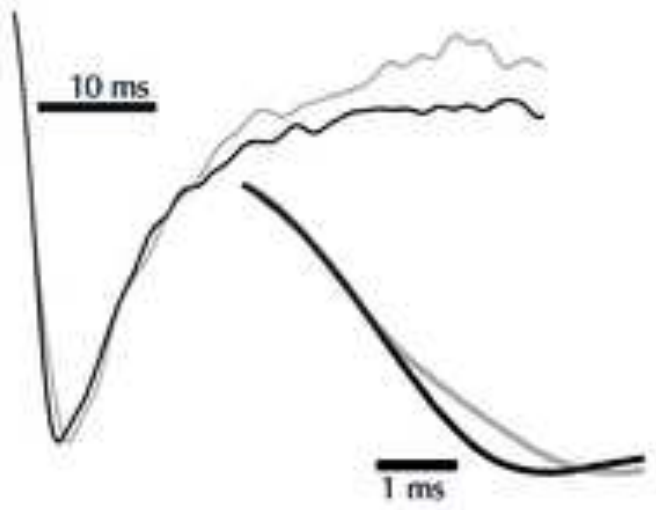



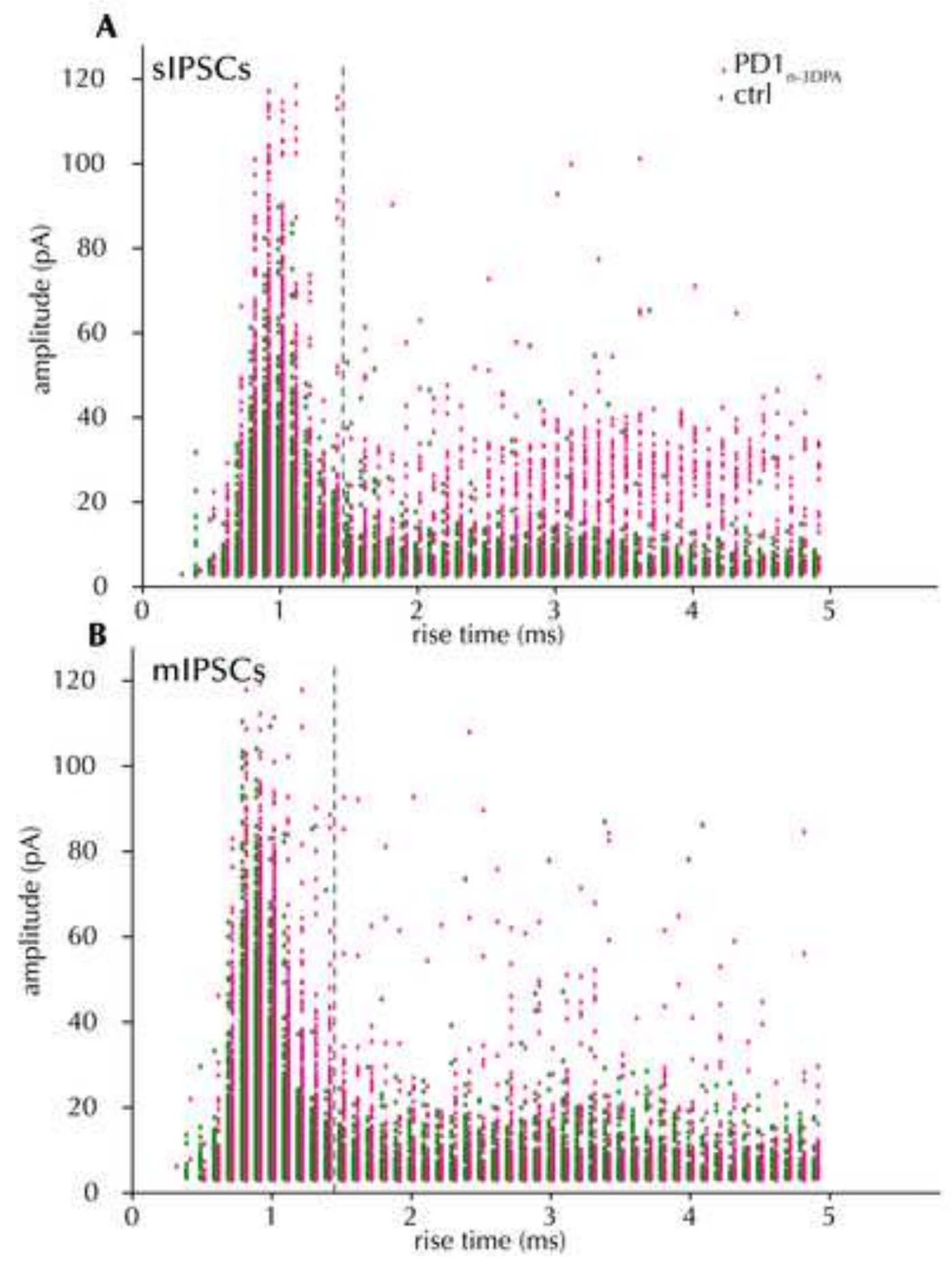
CA1

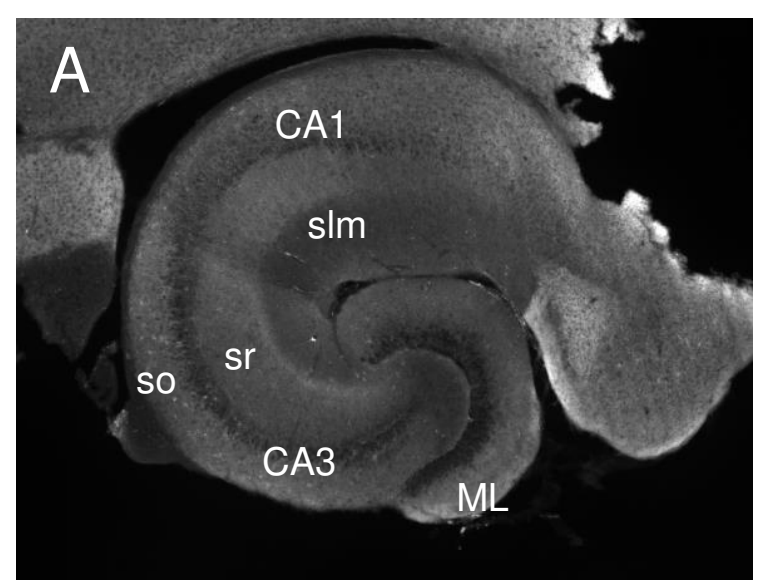

aCSF

PD1

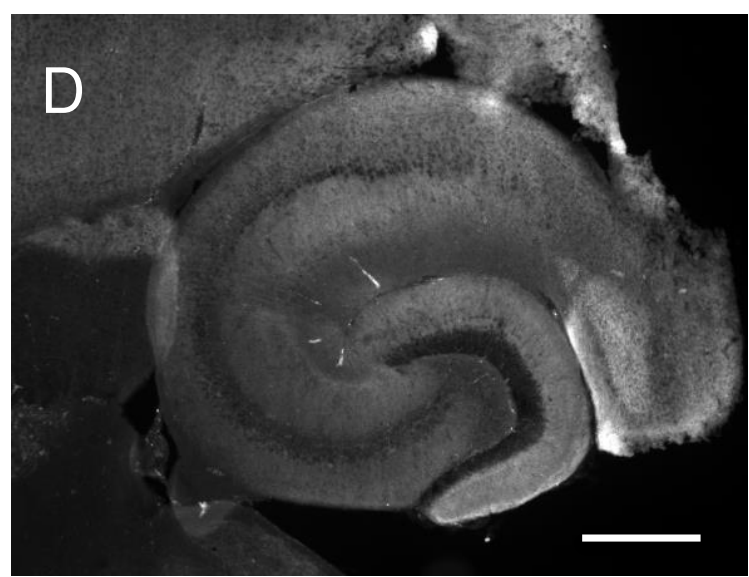

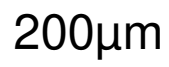

CA3
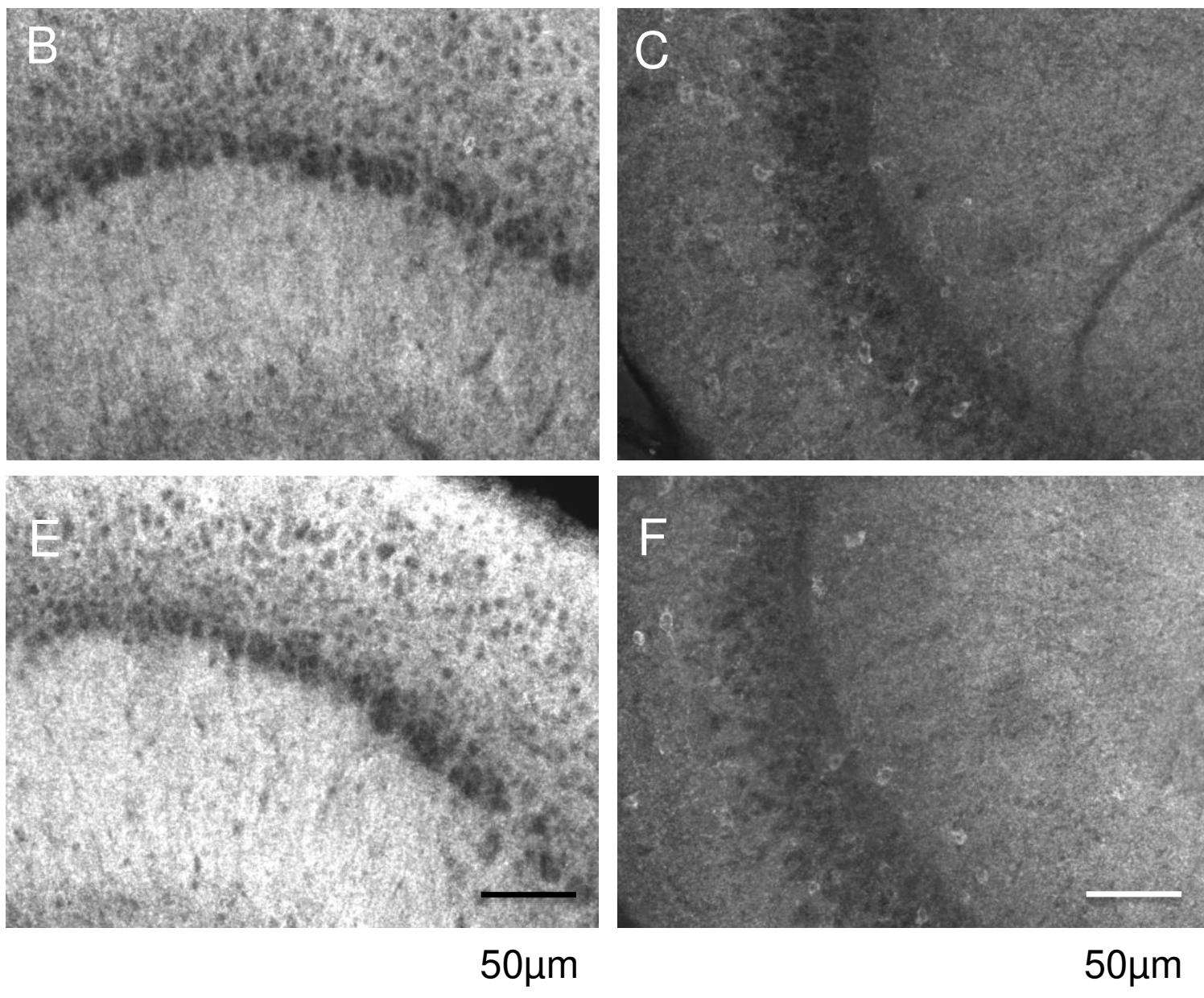
Figure 6

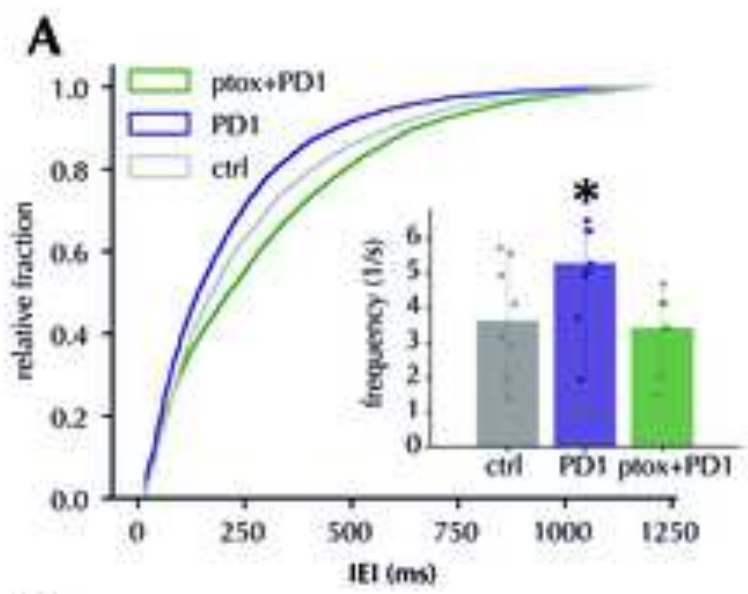

B
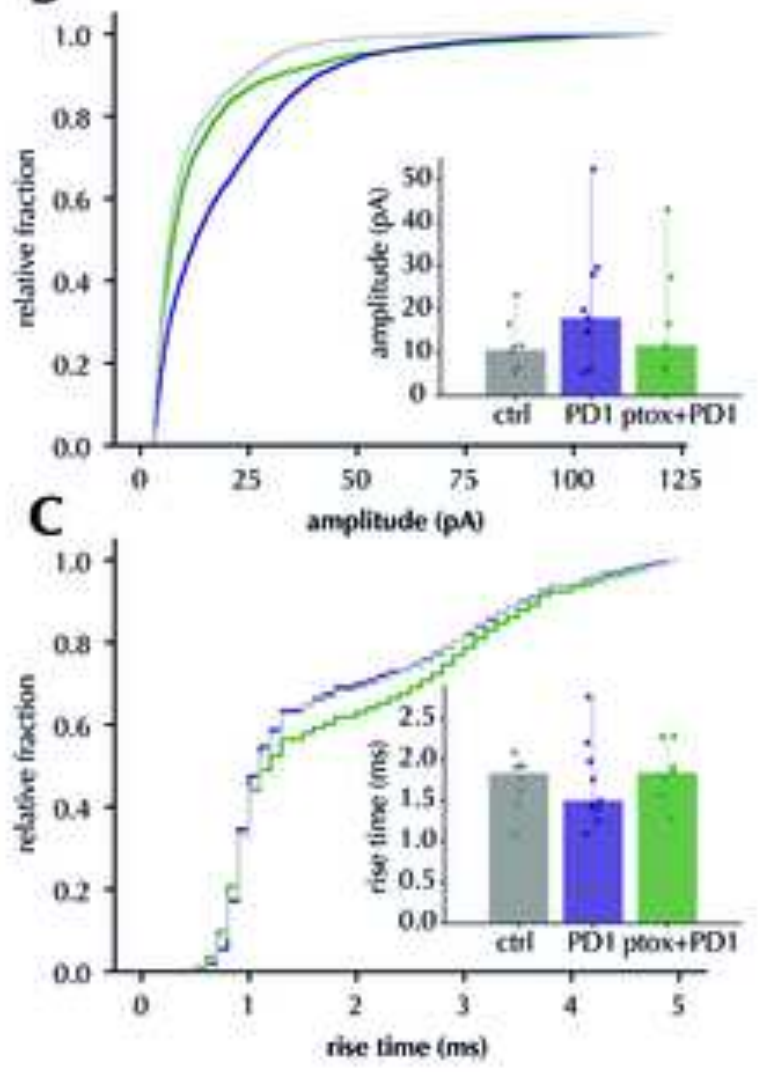